\title{
Intususcepción intestinal posterior a bypass gástrico en Y de Roux*
}

\author{
Drs. ENRIQUE NORERO M. ${ }^{1}$, ALEJANDRO RADDATZ E. ${ }^{1}$, SERGIO GUZMÁN B. ${ }^{1}$ \\ 1 Departamento de Cirugía Digestiva. División de Cirugía. \\ Facultad de Medicina. Pontificia Universidad Católica de Chile. Santiago, Chile.
}

\begin{abstract}
Intussusception after Roux-en-Y Gastric Bypass causing bowel obstruction. Report of one case
\end{abstract}

We report a 41 years old female who underwent a gastric bypass 10 years ago. She presented an excess weight loss of $114 \%$. She required a cholecystectomy and another surgical procedure due to an internal hernia. The patient complained of recurrent abdominal pain and an abdominal computed tomography showed an intestinal intussusception located at the jejuno-jejuno anastomosis. She was operated and the diagnosis was confirmed. An enlargement of the biliopancreatic loop and a bezoar were also found. A $15 \mathrm{~cm}$ intestinal resection of the enlarged portion of the biliopancreatic loop and a new jejuno-jejuno anastomosis were performed. The patient recovered uneventfully and was discharged on the fourth postoperative day. The occurrence of intestinal intussusception as a cause of intestinal obstruction after gastric bypass is rare. It has been reported after open and laparoscopic surgery. There are several theories about the cause of intussusceotion, like changes in intestinal motility, a high excess weight loss, but a conclusive cause has not been identified. Generally the location of the intussusceptions is at the jejuno-jejuno anastomosis and it appears to be in a retrograde fashion in the majority of cases. The treatment is controversial, with several options; simple reduction, reduction and intestinal fixation, but apparently intestinal resection has better results.

Key words: Gastric bypass, bowel obstruction, intestinal intussuscepction.

\section{Resumen}

Reportamos el caso de una paciente con antecedente de un bypass gástrico hace 10 años, que presentó una pérdida del exceso de peso de $114 \%$. Posteriormente se realizó una colecistectomía y cirugía por hernia interna. Debido a cuadros de dolor abdominal a repetición fue estudiada con una tomografía computada de abdomen que demostró una intususcepción intestinal a nivel de la entero-entero anastomosis. La paciente fue intervenida, se confirmó el diagnóstico y se encontró también una dilatación del asa biliopancreática y un bezoar. Se realizó una resección intestinal del asa biliopancreática de $15 \mathrm{cms}$ y anastomosis yeyuno-yeyunal, con buena evolución postoperatoria y alta a los 4 días de la cirugía. La intususcepción intestinal posterior a un bypass gástrico es poco frecuente como causa de obstrucción intestinal y ha sido descrita luego de cirugía

\footnotetext{
*Recibido el 24 de Agosto de 2009 y aceptado para publicación el 19 de Octubre de 2009.

Correspondencia: Dr. Enrique Norero M.

Marcoleta 367, Casilla 114-D, Santiago, Chile.

Fax: 5626329620

E-mail: enorero@puc.cl
} 
abierta y laparoscópica. No existe una explicación definitiva para su patogenia. Presenta algunas características particulares como producirse en la mayoría de los casos en relación a la entero-entero anastomosis y ser una intususcepción retrograda. Su tratamiento es controversial, existiendo varias alternativas; reducción simple, reducción y fijación del asa comprometida, pero aparentemente la resección intestinal tendría mejores resultados, con menor frecuencia de recurrencia.

Palabras clave: Bypass gástrico, obstrucción intestinal, intususcepción intestinal.

\section{Introducción}

La obesidad representa una verdadera epidemia a nivel mundial ${ }^{1}$. La cirugía bariátrica es el tratamiento con los mejores resultados a largo plazo $^{2}$. El bypass gástrico presenta buenos resultados, sin embargo, no está exento de morbilidad tanto a corto como a largo plazo $^{3}$. Un grupo de pacientes puede presentar obstrucción intestinal en el seguimiento, secundario a hernias internas o bridas ${ }^{4}$. Una causa rara descrita de obstrucción intestinal posterior a bypass gástrico es la intususcepción intestinal. Reportamos el caso de una paciente de sexo femenino con antecedente de bypass gástrico que presentó una obstrucción intestinal debido a una intususcepción intestinal.

\section{Caso clínico}

Paciente de sexo femenino, de 41 años de edad, con antecedentes de insuficiencia suprarrenal congénita bajo tratamiento, síndrome depresivo mayor y tabaquismo crónico. Luego de evaluación multidisciplinaria fue operada hace 10 años atrás por obe- sidad mórbida (IMC 44, peso $96 \mathrm{~kg}$ ), realizándose un bypass gástrico abierto en Y de Roux, con bolsa gástrica de $15-20 \mathrm{cc}$ y asa alimentaria de $150 \mathrm{~cm}$ de longitud ascendida por vía transmesocólica.

La paciente es controlada en forma ambulatoria; a los 9 meses presentaba una pérdida del exceso de peso de $71 \%$ (peso de $66 \mathrm{~kg}$ ); se diagnosticó colelitiasis por lo que se realiza una colecistectomía abierta.

Logra en forma estable una pérdida del exceso de peso de $123 \%$ (IMC 20, peso de $44 \mathrm{~kg}$ ). Se mantiene en controles periódicos.

A los 10,5 años del bypass gástrico, debido a repetidos cuadros de dolor abdominal e imagen compatible con hernia interna, se realiza laparotomía identificándose una hernia interna en el mesocolon transverso con ascenso del asa biliopancreática la cual está dilatada. Se reduce el asa y se cierra el orificio del mesocolon, con buena evolución postoperatoria.

A los 11 años del bypass gástrico la paciente presenta nuevamente cuadros de dolor abdominal a repetición, asociado a vómitos y náuseas por los que se estudia con tomografía computada de abdomen encontrándose una imagen de intususcepción en re-
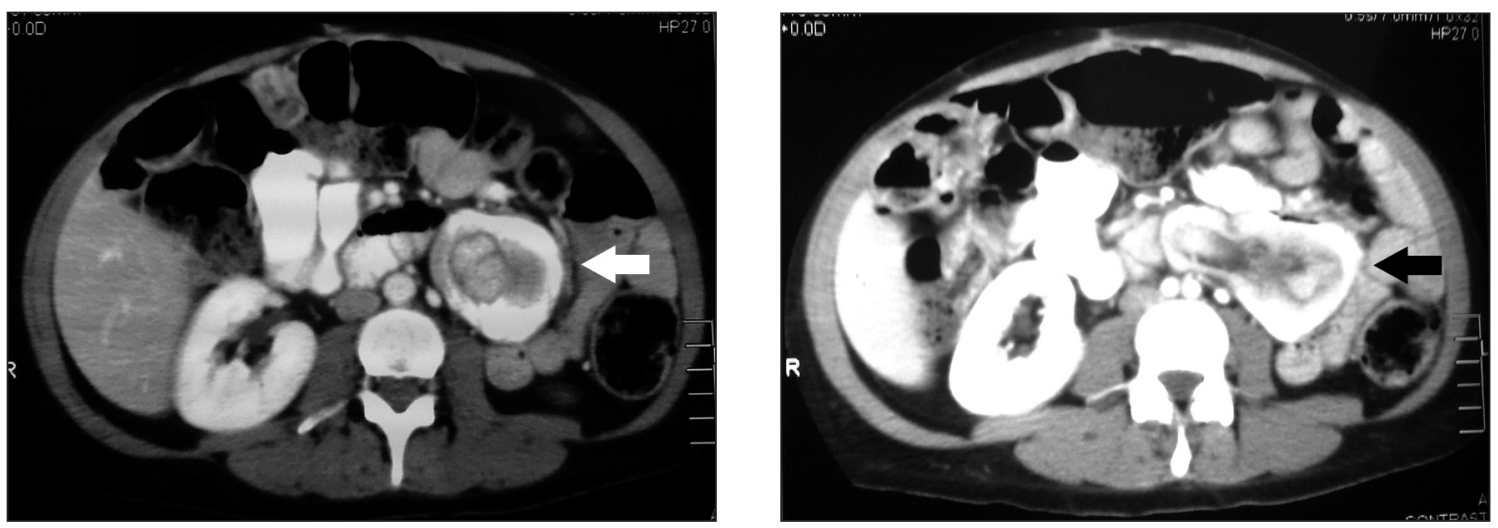

Figura 1. Tomografia computada de abdomen que muestra intususcepción intestinal a nivel de la entero-entero anastomosis (flecha). 

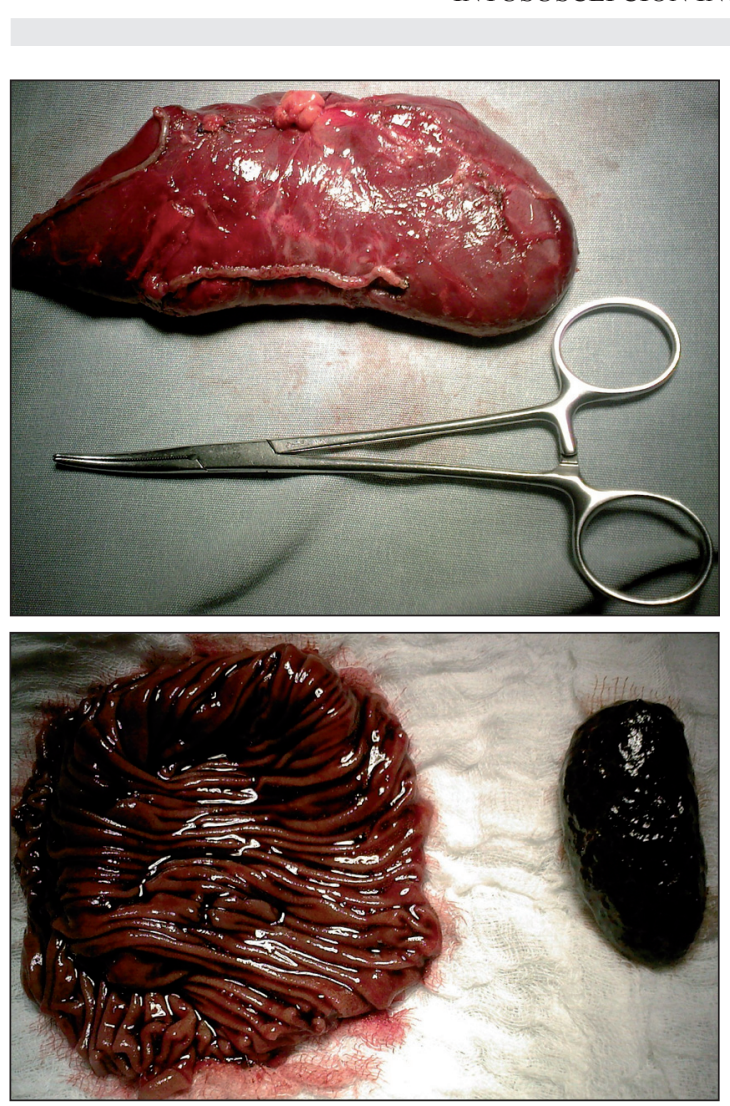

Figura 2. Asa biliopancreática dilatada que fue resecada y bezoar.

lación a la entero-entero anastomosis (Figura 1). Se realiza laparotomía, confirmando este diagnóstico, encontrándose también una importante dilatación del asa biliopancreática y un bezoar proximal a la entero-entero anastomosis. No hay hernias internas en esta laparotomía. Se resecan $15 \mathrm{~cm}$ del asa biliopancreatica y se rehace la anastomosis yeyunoyeyunal manual, fijando el asa biliopancreática a la alimentaria (Figura 2). La paciente presenta buena evolución postoperatoria, siendo dada de alta el día 4 postoperatorio.

\section{Discusión}

El bypass gástrico es una de las técnicas de cirugía bariátrica más difundidas en el mundo, con buenos resultados a largo plazo. Sin embargo, no está exento de morbilidad, destacando a corto plazo la filtración anastomótica y/o de la bolsa gástrica, estenosis de la anastomosis gastro-yeyunal ${ }^{3}$ y a largo plazo la obstrucción intestinal ${ }^{4}$, así como los déficit nutricionales a vitaminas, calcio, vitamina B12, entre otros.
La obstrucción intestinal post bypass gástrico se puede deber a bridas o a hernias internas, a nivel del mesocolon, brecha del meso del intestino delgado o espacio de Petersen ${ }^{5}$. En nuestro centro se ha reportado previamente una cifra de obstrucción intestinal de $4 \%$ para bypass gástrico laparoscópico ${ }^{4}$ y este caso, representa el primer reporte de una intususcepción post bypass gástrico en nuestro centro en más de 15 años de experiencia en cirugía bariátrica.

La primera comunicación de intususcepción posterior a bypass gástrico data de $1991^{6}$. Desde entonces se han comunicado alrededor de 50 casos, la mayoría durante los últimos 5 años, representando una causa rara de obstrucción intestinal post bypass gástrico $^{5,7-19}$. Se ha descrito tanto como cuadro agudo como subagudo con episodios de dolor abdominal recurrente. En algunos casos se ha descrito necrosis y perforación del asa ${ }^{6,8,11}$.

La intususcepción posterior al bypass gástrico presenta algunas particularidades, como producirse en la gran mayoría de casos a nivel de la enteroentero anastomosis. En segundo lugar es típicamente de tipo retrogrado, o sea el asa distal se intususcepta en un asa proximal. Estas particularidades no parecieran ser diferentes en los casos ocurridos con posterioridad a cirugía abierta y laparoscópica ${ }^{7-9,11,16,17,19}$.

La mayoría de los casos de intususcepción intestinal primaria se producen en población pediátrica. En adultos es menos frecuente y generalmente se identifica alguna causa que lo favorece, como un tumor intestinal, enfermedad inflamatoria y divertículo de Meckel, entre otras ${ }^{20-22}$. En la situación de los pacientes sometidos a bypass gástrico se han planteado varias teorías, como alteraciones de la motilidad intestinal debido a la aparición de marcapasos anómalos con producción de ondas peristálticas que podrían llegar a la anastomosis yeyuno-yeyunal en forma simultánea creando una onda de mayor amplitud $^{6,14,16}$. Otra teoría es que las líneas de corchetes podrían actuar como punto de tracción ${ }^{14}$. También se ha planteado que la baja excesiva de peso disminuye la tracción de los mesos y de esta manera el intestino estaría más propenso a esta condición ${ }^{16}$; en nuestro caso la paciente tuvo una pérdida del exceso de peso de $114 \%$, lo que podría haber favorecido esta situación. También creemos que la dilatación del asa biliopancreática que presentaba la paciente, favorecido por la hernia interna por la cual fue intervenida meses atrás, puede haber favorecido la intususcepción. Sin embargo, siendo esta una complicación infrecuente no hay una causa definitiva ${ }^{5,14,16}$.

El tratamiento es quirúrgico. La presencia de necrosis del asa o perforación es indicación de resección intestinal ${ }^{6,8,11}$. En los cuadros en que se logra reducir el asa y ésta se encuentra vital, existe controversia sobre la mejor alternativa; simple reducción, 
fijación del intestino o resección intestinal. En la mayor experiencia comunicada de esta patología que incluye 23 casos por el grupo de Salt Lake City, se encontró que la tasa de recurrencia era menor con una resección intestinal ${ }^{11}$. En este caso, a pesar de la vitalidad del intestino, se optó por esta técnica, con el objetivo de resecar el asa biliopancreática dilatada, sin complicaciones en el postoperatorio inmediato.

\section{Referencias}

1. Klein S, Wadden T, Sugermann HJ. AGA technical review on obesity. Gastroenterology 2002; 123: 882-932.

2. Christou N, Sampalis J, Liberman M, Look D, Auger $\mathrm{S}$, Mclean A, et al. Surgery decreases long-term mortality, morbidity and health care use in morbidly obese patients. Ann Surg 2004; 240: 416-424.

3. Escalona A. Complicaciones quirúrgicas del bypass gástrico laparoscópico. Rev Chil Cir 2006, 58: 97-105.

4. Escalona A, Devaud, Pérez G, Crovari F, Boza C, Viviani $\mathrm{P}$, et al. Antecolic versus retrocolic alimentary limb in laparoscopic Roux-en-Y gastric bypass: a comparative study. Surg Obes Relat Dis 2007; 3: 423-427.

5. Edwards M, Grinbaum R, Ellsmere J, Jones D, Schneider B. Intussusception after Roux-en-Y gastric bypass for morbid obesity: case report and literature review of rare complication. Surg Obes Relat Dis 2006; 2: 483489.

6. Hocking M, Mccoy D, Vogel S, Kaude J, Sninsky C. Antiperistaltic and isoperistaltic intussusception associated with abnormal motility after Roux-en-Y gastric bypass: a case report. Surgery 1991; 110: 109-112.

7. Kasotakis G, Sudan R. Retrograde intussusception after Roux-en-Y gastric bypass for morbid obesity. Obes Surg 2009; 19: 381-384.

8. Efthimiou E, Court O, Christou N. Small bowel obstruction due to retrograde intussusceptions after laparoscopic Roux-en-Y gastric bypass. Obes Surg 2009; 19: 378-380.

9. Pauli E, Haluck R. Antiperistaltic (retrograde) intussusceptions after laparoscopic Roux-en-Y gastric bypass procedure. Surg Obes Relat Dis 2008; 4: 567-568.

10. Lessmann J, Soto E, Merola S. Intussusceptions after
Roux-en-Y gastric bypass for morbid obesity. Surg Obes Relat Dis 2008; 4: 664-667.

11. Simper S, Erzinger J, Mckinlay R, Smith S. Retrograde (reverse) jejunal intussusceptions might not be such a rare problem: a single group's experience of 23 cases. Surg Obes Relat Dis 2008; 4: 77-83.

12. Al-Sabah S, Christou N. Intussusceptions after laparoscopic Roux-en-Y gastric bypass. Surg Obes Relat Dis 2008; 4: 205-209.

13. Zainabadi K, Ramanathan R. Intussusceptions after laparoscopic Roux-en-Y gastric bypass. Surg Obes 2007; 17: 1619-1623.

14. Coster D, Sundberg S, Kermode D, Beitzel D, Noun S, Severidt M. Small bowel obstruction due to antegrade and retrograde intussusceptions after gastric bypass: three case reports in two patients, literature review, and recommendations for diagnosis and treatment. Surg Obes Relat Dis 2008; 4: 69-72.

15. Wax J, Wolff R, Cobean R, Pinette M, Blackstone J, Cartin A. Intussuscepstion complicating pregnancy following laparoscopic Roux-en-Y gastric bypass. Obes Surg 2007; 17: 977-979.

16. Ver Steeg K. Retrograde intussusception following Roux-en-Y gastric bypass. Obes Surg 2006; 16: 11011103.

17. Majeski J, Fried D. Retrograde intussusception after Roux-en-Y gastric bypass surgery. J Am Coll Surg 2004; 1999; 988-989.

18. Papadia F. Intussusception after gastric bypass. Obes Surg 2004; 14: 705.

19. Bocker J, Vasile J, Zager J, Goodman E. Intussusception: an uncommon cause of postoperative small bowell obstruction after gastric bypass. Obes Surg 2004; 14 : 116-119.

20. Goverman J, Greenwald M, Gellman L, Gadaleta D. Antiperistaltic (retrograde) intussusception after Rouxen-Y gastric bypass. Am Surg 2004; 70: 67-70.

21. Duane $\mathrm{T}$, Wohlgemuth $\mathrm{S}$, Ruffin K. Intussusception after Roux-en-Y gastric bypass. Am Surg 2000; 66: 82-84.

22. Marinis A, Yiallourou A, Samanides L, Dafnios N, Anastasopoulos G, Vassiliou I, et al. Intussusception of the bowel in adults: a review. World J Gastroenterol 2009; 15: 407-411. 